\title{
Hyperglycemia Presenting with Hemichorea-Hemiballismus and T-1 Hyperintensity on MRI Brain
}

\author{
Saima Shafait ${ }^{1}$, Wasim Alamgir ${ }^{2}$ and Mobeen Shafique ${ }^{3}$ \\ ${ }^{1}$ Department of Neurology, Pakistan Emirates Military Hospital, Rawalpindi, Pakistan \\ ${ }^{2}$ Department of Neurology, Army Medical College, Rawalpindi, Pakistan \\ ${ }^{3}$ Department of Radiology, Armed Forces Institute of Radiology and Imaging, Rawalpindi, Pakistan
}

\begin{abstract}
Hemichorea-hemiballismus, secondary to hyperglycemia, is a rare but easily treatable condition that is usually associated with type II diabetes mellitus. This is a case of a 68- year lady, with long-standing, poorly controlled diabetes mellitus, who presented with disabling right-sided hemichorea-hemiballismus. The T1-weighted magnetic resonance imaging revealed hyperintensity in the basal ganglia. The abnormal movements subsided within a few days after achieving euglycaemia with insulin therapy. This case highlights the importance of treatment of hyperglycaemia in a diabetic patient presenting with acute or sub-acute abnormal movement disorder.
\end{abstract}

Key Words: Hemichorea, Hemiballismus, Hyperglycemia, T-1 hyperintensity.

How to cite this article: Shafait S, Alamgir W, Shafique M. Hyperglycemia Presenting with Hemichorea-Hemiballismus and T-1 Hyperintensity on MRI Brain. J Coll Physicians Surg Pak 2021; 31(10):1228-1230.

\section{INTRODUCTION}

Hemichorea-hemiballismus is a rare hyperkinetic movement disorder characterised by continuous involuntary movement of one or both limbs on one side of the body. ${ }^{1}$ Hemichorea-hemiballismus with hyperglycemia was first reported in 1960 by Bedwell; however, the classic triad of hemichorea-hemiballismus, hyperglycemia, and T1 hyperintensity was described in 1994 by Yahikozawa et al. It usually presents after recent episodes of hyperglycemia; however, it may present few weeks after blood glucose is controlled and actively being treated. ${ }^{2}$ Brain magnetic resonance imaging (MRI) is very important in assessment of such patients. We, hereby, report a case of a 68 -year female who was known diabetic and presented with subacute onset hemichorea-hemiballismus after weeks of poorly controlled diabetes and non-compliance with her oral hypoglycemic drugs.

\section{CASE REPORT}

A 68-year female presented to emergency department of our hospital with continuous non-rhythmic involuntary movements of right side of her body.

Correspondence to: Dr. Saima Shafait, Department of Neurology, Pakistan Emirates Military Hospital,

Rawalpindi, Pakistan

E-mail: saima_shafait@hotmail.com

Received: November 14, 2019; Revised: January 28, 2020;

Accepted: March 12, 2020

DOI: https://doi.org/10.29271/jcpsp.2021.10.1228
The symptoms started a week before the presentation, initially involving right lower limb with subsequent progression to right upper limb and face. The movements significantly interfered with her daily activities and resolved during the sleep.The patient or her family did not report any concomitant personality change or cognitive deficit. Her past medical history included poorly controlled type II diabetes mellitus, diagnosed 13 years ago, treated with oral hypoglycemic drugs with poor compliance. She denied use of any other prescribed or over-the-counter medication, or herbal supplements.

The neurological examination revealed involuntary, high amplitude, non-rhythmic movements, over the proximal muscle groups of right upper and lower limbs. The distal muscle groups of the right upper and lower limbs as well as the right side of the face showed involuntary, low amplitude movements with a flowing quality. The rest of the physical examination did not showany significant findings.

Initial laboratory workup revealed serum glucose levels of 468 $\mathrm{mg} / \mathrm{dL}$ and the hemoglobin $\mathrm{A} 1 \mathrm{C}$ of $14.1 \%$. Renal functions, liver and thyroid function tests, serum electrolytes ( $\mathrm{Na}, \mathrm{K}, \mathrm{Ca}, \mathrm{Mg}$, and PO4), and parathyroid hormone levels were within the normal range. Urine analysis showed significant glycosuria with no ketones. Computed tomographic (CT) scan of brain showed left basal ganglia hyperdensity with no associated mass effect, edema, or volume loss. MRI brain showed high signal intensity over left basal ganglia on T1-weighted sequence which appeared hypointense on T2-weighted sequences. Hemorrhage was ruled out by the absence of susceptibility on gradient 
eco (GRE). There were few discrete multifocal T2 flair hyperintensities in the sub-cortical and peri-ventricular region likely representing ischemic foci (Figure 1 ).
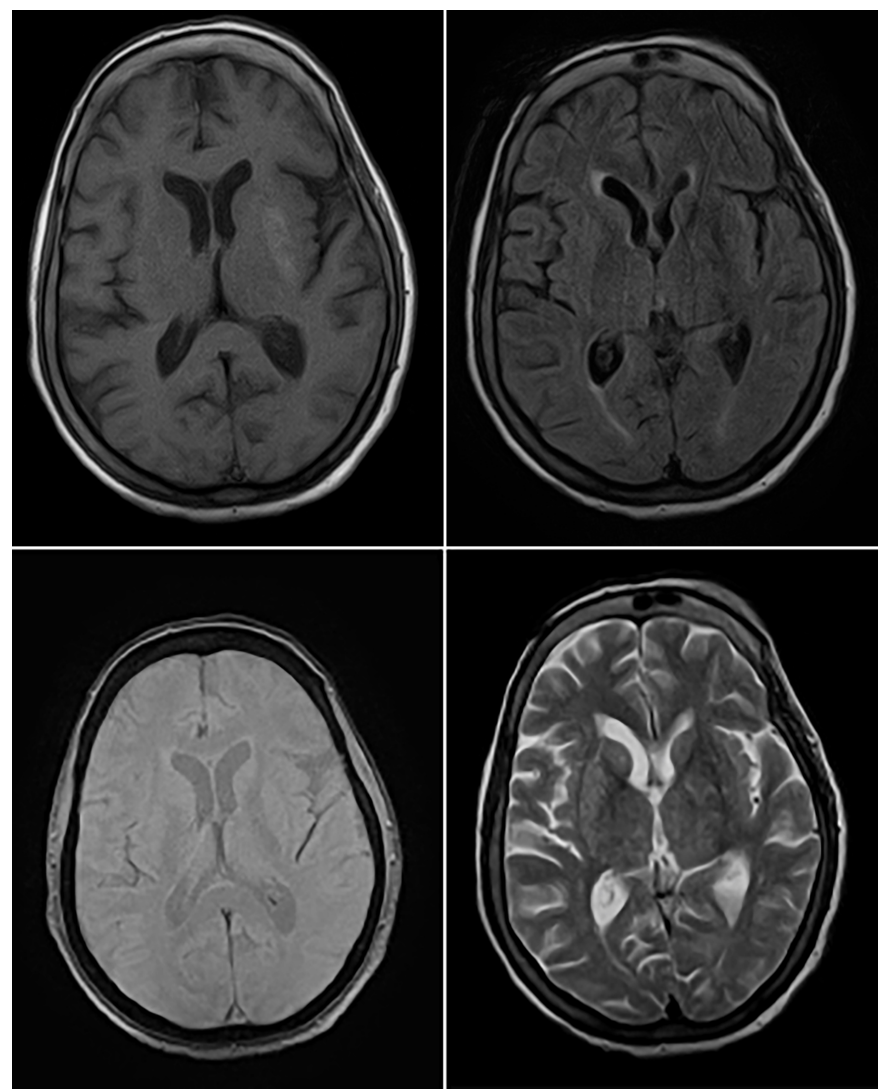

Figure 1: Magnetic resonance image scan of the patient showing left putamen hyperintensity (top left), corresponding hypointensity on FLAIR (top right) and T2 (bottom right) sequences. GRE (bottom left) showed no evidence of susceptibility.

Strict blood glucose control was achieved with target blood glucose of $80-120 \mathrm{mg} / \mathrm{dl}$ using long-acting insulin (Lantus) at night and short-acting insulin, three times a day, with each meal. Sodium valproate and haloperidol were used for control of involuntary movements. The abnormal body movements responded promptly to aggressive glycaemic control and by day 5 of hospital stay, she was able to perform most of her daily activities independently with active participation in physical therapy exercises. She was discharged on insulin, metformin, haloperidol and pregabalin. At the follow-up visit, 3 months later, she had near complete resolution of the involuntary movements of her extremities.

\section{DISCUSSION}

Hemichorea-hemiballismus with non-ketotic hyperglycemia, also called diabetic striatopathy, is a well-recognised syndrome characterised by sudden onset of hemichorea or its more severe expression, hemiballismus. It typically affects older females with poorly controlled typell diabetes. Estimated prevalence of this disorder is less than 1 in 100,000 people with female to male ratio of 1.8:1 and mean age of onset of 70 years. Most of the cases have been described in individuals of Asian descent likely suggesting genetic predisposition.
Hemichorea-hemiballismus can be the first presentation of diabetes mellitus or may occur after prolonged period of poor glycemic control. Neuroimaging typically shows hyperintense lesions in basal ganglia on T1-weighted MRI brain. Clinical and imaging findings are typically unilateral; although, they can be bilateral in up to $11.4 \%$ cases. $^{3}$ The condition is potentially reversible usually resolving within 2 to 12 months after treatmentofhyperglycemia. ${ }^{4}$ Case reports from currentmedicalliterature have shown that patients show dramatic recovery from their hyperkinesia with aggressive serum glucose control. Female gender, extraputaminal lesions and delayed introduction of dopamine receptor blocking agents have been associated with poor prognosis. ${ }^{5}$ However, tardive dyskinesias may develop secondary to dopamine receptor blocking agents as diabetics are already more sensitive to neuroleptics because of microcirculatory changes of diabetes mellitus.

The differential diagnoses of hemichorea-hemiballismusare quite vast, including ischemic or hemorrhagic stroke, vasculitis, central nervous system lupus, mass lesion, multiple sclerosis, thyrotoxicosis, and drugs (such as neuroleptics or levodopa). In contrast, the causes of increased T1 signal intensity in the basal ganglia are rare, such as basal ganglia calcification, Tay-Sachs disease, tuberous sclerosis, chronic hepatic encephalopathy, manganese toxicity, Wilson disease, post-cardiac arrest encephalopathy, hypoglycemic coma, hypothyroidism, neurofibromatosis, Fahr disease and carbon monoxide poisoning. ${ }^{6}$ However, unlike in our case, in many of these conditions, the lesions are either symmetrical or more widespread.

The pathophysiology of diabetics striatopathy is not completely understood; however, metabolic changes secondary to blood brain barrier disruption might play a role. The hyperglycemic state induces anaerobic metabolic pathways whereby inhibitory neurotransmitters such as acetylcholinesterase and gamma-amino-butyric acid (GABA) are used as an alternative source of energy. The resultant drop in GABA and acetate levels leads to decreased acetylcholine synthesis causing a dysfunction of basal ganglia which clinically manifests as involuntary movements (chorea-hemiballismus). ${ }^{7,8}$ PET and SPECT scans done in such cases have been inconclusive, suggesting that altered glucose metabolism might not be the key factor in development of hemichorea-hemiballismus; rather histological changes like cellular ischemia and gliosis are more important in the pathogenesis of this condition. ${ }^{9}$ An autopsy report of a 92year male presenting with the classic triad showed multiple foci of recent infarcts associated with reactive astrocytic and interneuronal response. ${ }^{10}$

Hemichorea-hemiballismus with non-ketotic hyperglycemia is a rare but treatable condition, associated with poorly controlled diabetes. Prompt recognition and treatment is essential to avoid unnecessary delay in management and to prevent adverse outcomes.

\section{PATIENT'S CONSENT:}

Informed consent was obtained from the patient for publication of the case report. 


\section{CONFLICT OF INTEREST:}

The authors declared no conflict of interest.

\section{AUTHORS' CONTRIBUTION:}

SS: Data acquisition, interpretation, drafting, integrity of work. WA: Design and conception, critical review and comment of manuscript, approval of the final version of the manuscript MS: Data acquisition, critical review and comment of manuscript.

\section{REFERENCES}

1. Ozgur A, Esen K, Kaleagasi H, Yilmaz A, Kara E. Diabetic striatopathy in a patient with hemiballism. EmergRadiol 2015; 22(3):347-9. doi: 10.1007/s10140-015-1308-7.

2. Sperling M, Bhowansingh R. Chorea hyperglycemia basal ganglia syndrome in a 63-year-old Male. Hindawi 2018; 2018:9101207. doi: 10.1155/2018/9101207.

3. Krishna S, Sodhi KS, Saxena AK, Singhi P, Khandelwal N. Hyperdense basal ganglia in nonketotic hyperglycemia. J Emerg Med 2015; 49(2):57-8. doi: 10.1016/j.jemermed. 2015.02.016.

4. Chokshi FH, Aygun N, Mullins ME. Imaging of acquired metabolic and toxic disorders of the basal ganglia. Semin Ultrasound CT MR 2014; 35(2):75-84. doi: 10.1053/j.sult.
2013.09.006.

5. Fong SL, Tan AH, Lau KF, Ramli N, Lim S. Hyperglycemiaassociated hemichorea-hemiballismus with predominant ipsilateral putaminal abnormality on neuroimaging. J Mov Disord 2019; 12(3):187-9. doi: 10.14802/jmd.19014.

6. Lai PH, Tien RD, Chang MH, Teng MM, Yang CF, Pan HB, et al. Chorea-ballismus with nonketotic hyperglycemia in primary diabetes mellitus. Am J Neuroradiol 1996; 17(6):1057-64.

7. Aggarwal A, Bansal N, Aggarwal R. Nonketotic hyperglycemia presenting as monoballism. J Emerg Med 2016; 50(3):e133e4. doi: 10.1016/j.jemermed.2015.11.016.

8. Hansford BG, Albert D, Yang E. Classic neuroimaging findings of nonketotic hyperglycemia on computed tomography and magnetic resonance imaging with absence of typical movement disorder symptoms (hemichorea- hemiballismus). J Radiol Case Rep 2013; 7(8):1-9. doi: 10.3941/jrcr.v7i8.1470.

9. Gomez Ochoa SA, Espin Chicho. Understanding the pathophysiology of hyperglycemia-associated chorea-ballism: A systematic review of positron emission tomography findings. Funct Neurol 2018; 33(2):67-72.

10. Ohara S, Nakagawa S, Tabata K, Hashimoto T. Hemiballismus with hyperglycemia and striatal T1-MRI hyperintensity: An autopsy report. Mov Disord 2001; 16(3):521-5. doi: 10.1002/ mds.1110. 